\title{
El morfema -ku en el Quechua Ancashino
}

\author{
The -ku morpheme in the Ancash Quechua
}

\author{
Francisco Carranza Romero ${ }^{1}$
}

\section{RESUMEN}

Describe y analiza las diferentes realizaciones morfosemánticas del sufijo -ku en el quechua ancashino. La presencia del sufijo - ku en el quechua Ancashino es abundante, además cumple diferentes funciones gramaticales y, consecuentemente, adquiere diferentes significados. La data analizada corresponde a los datos empíricos propios acopiados en varios años de investigación en las diferentes áreas que comprende el quechua ancashino. Se aborda el estudio desde la perspectiva de la lingüística descriptiva andina y se concluye puntualizando que el variado uso del sufijo - ku enriquece el discurso quechua; pero, al mismo tiempo, crea dificultades a los que aprenden el quechua como segunda lengua.

Palabras clave: sufijo -ku, quechua ancashino; funciones gramaticales; morfosemnántica.

\section{ABSTRACT}

This article describes and analyzes the different morphosemantic realizations of the suffix - ku in Ancash Quechua. The presence of the -ku suffix in Ancash Quechua is abundant, besides fulfilling different grammatical functions and, consequently, acquires different meanings. The data analyzed correspond to the empirical data collected in several years of research in the different areas that comprise the Ancash Quechua. The study is approached from the perspective of Andean descriptive linguistics and it is concluded that the varied use of the $-k u$ suffix enriches the Quechua discourse; but, at the same time, it creates difficulties for those who learn Quechua as a second language.

Keywords: suffix -ku, ancash quechua; grammatical functions; morphosemantic.

1 Instituto de Estudios de Asia y América, «Universidad Dankook», Seúl, Corea. 


\section{ICHIKLLACHAW}

Kay musyapakuyqa imanaw Anqash qichwachaw sufihu -keu tukuy laaya kanqantam willakamun. Kay sufihu -ku nishqan Anqash qichwachawqa atskam, hina tukuy laayamanmi tikran, hinaman tukuy laayaman niqninkuna tikran. Kay musyapakuy rurayqa kikin rimaqkunapitam atska watapiqnam ashir ashir ayllukashqa, Anqash suyuchawchaw llapan qichwa rimaqkunapita. Kay musyapakuyqa rurakashqa linguistika discriptiba antichaw kanqanpitam, ichik shimichaw kayta nishwan sufihu -ku, kayqa qichwa shimintsiktam kallpayaatsin, hinaman hukpiqqa qichwa yachakuqkunatam yachakuyninkunachaw sasayaatsin ishkay kaq shimina kanqanraykur.

Pushaq shimikuna: ushaq pakishimi -ku; anqash qichwa; gramatika ruraynin; morfosemantika.

\section{INTRODUCCIÓN}

La lengua quechua expresa las variaciones morfosemánticas agregando una o más partículas a una raíz o base. «El Quechua es una lengua aglutinante de tipo sufijante, lo cual quiere decir que las palabras se forman mediante la adición a la raíz de un conjunto de pequeñas partículas llamadas sufijos» (Julca, 2009: 52).

La presencia del morfema - ku es abundante en los enunciados del quechua ancashino (Quechua I); su uso, combinación y distribución hacen cambiar el contenido del mensaje. Una advertencia: Transcribo conscientemente en todos mis trabajos la semiconsonante w en posición silábica prenuclear de los diptongos wa, wi (waka = adoratorio; wiyay $=$ oír); pero, al margen de la norma ortográfica quechua, prefiero transcribir $u$ a la semivocal en el margen final de las sílabas en los diptongos au, yu (waychau = huaychao (pájaro de la puna), yukris = zorzal; los sufijos - chau = en, - nau = como). Que me disculpen los académicos quechuas. El aprendizaje del alfabeto latino por los quechuas se complica cuando los alfabetos usados en el quechua y el castellano se distancian. Ya es bastante el uso de la grafía h (hache) en el quechua en vez de la grafía j (jota) del castellano.

Otras advertencias sobre este trabajo: 1) Para diferenciar el quechua y el castellano recurrimos a las letras cursivas para el quechua; las normales para el castellano. 2) Las siglas: $\mathrm{P}=$ Pregunta; $\mathrm{R}=$ Respuesta; morf. $=$ morfema.

\section{MATERIALES Y MÉTODOS}

El universo de estudio comprendió la zona andina de Áncash. Para el acopio de datos se ha utilizado varias técnicas e instrumentos como la entrevista aplicada a los lugareños de los pueblos visitados, así como la revisión de materiales grabados y escritos de quechuahablantes. El trabajo de investigación se enmarcó en los casos de los estudios lingüísticos descriptivos debido a que el propósito principal fue pesquisar y describir de una manera pormenorizada las funciones gramaticales y semánticas del morfema $-k u$ en el quechua ancashino. 


\section{RESULTADOS Y DISCUSIÓN}

1. $-k u$, interrogativo, disyuntivo, hipocorístico

\subsection{Morfema Interrogativo}

Este morfema - ku (-chu en Quechua II) es ubicado en el núcleo de la interrogación. Esta distribución ayuda a entender mejor el mensaje porque funciona también como topicalizador. Por esta razón, en la respuesta también se usa el sufijo topicalizador en donde fue el núcleo de la pregunta.

Núcleo nombre:

P. ¿Shantiku yanapamanqa?

R. Manam, Shantiqa yanapashunkitsu.

P. ¿Santiago me ayudará?

R. No, Santiago no te ayudará.

El nombre propio Shanti (hipocorístico de Santiago) tiene dos morfemas que también funcionan como topicalizadores: -ku en la interrogación; -qa en la respuesta.

P. ¿Ayaku qupikun?

R. Ari, ayam qupikun.

P. ¿El alma aplasta?

R. Sí, el alma aplasta.

El nombre común aya (alma) tiene dos morfemas: -ku interrogativo; - $m$ afirmativo topicalizador en la respuesta. El morfma -m (en distribución postvocálica simple) tiene su alomorfo - mi (en distribución postvocálica larga y postconsonántica).

Aya qupikun (qupi-kun-n) = El alma aplasta. Es la creencia de que el espíritu errante del muerto se posesiona del cuerpo de un humano vivo para absorber su vitalidad física. Los que no creen relacionan este hecho con la pesadilla (opresión en el pecho del que está durmiendo).

Núcleo adjetivo:

P. ¿Hatunku markayki?

R. Ari, hatunmi markaa.

P. ¿Es grande tu pueblo?

R. Sí, es grande mi pueblo.

El adjetivo hatun (grande) de la respuesta lleva el morfema -mi por terminar en consonante. 
Núcleo adverbio:

P. ¿Qanyanku chaamurqayki?

R. Ari, qanyanmi chaamurqaa.

P. ¿Fue ayer que llegaste? (¿Llegaste ayer?)

R. Sí, ayer llegué. (Sí llegué ayer y no otro día).

Aquí también el adverbio qanyan (ayer) de la respuesta, lleva el morfema -mi por terminar en consonante.

Núcleo verbo:

P. ¿Shantiqa yanapamanqaku?

R. Shantiqa yanapashunkitsu.

P. ¿Me ayudará Santiago? (Dudo que Santiago me ayude)

R. Santiago no te ayudará.

Como el castellano carece del morfema sufijal interrogativo, en la traducción se puede solucionar cambiando el orden del sintagma oracional: el núcleo de la interrogación precediendo a otros elementos).

Como observamos en el diálogo superior, aparecen dos sufijos en la interrogación: Shanti-qa, topicalizador en el nombre; yanapamanqa-ku, interrogativo en el verbo conjugado. En la respuesta también hay dos morfemas: -qa en el nombre; -tsu negativo en el verbo. Sin embargo, el morfema interrogativo -ku (-chu en Quechua II) no aparece cuando se usan las palabras interrogativas o exclamativas como los pronombres $p i$ (quién\}, ima (qué); los adverbios may (dónde), imay (cuando), ayka (cuánto).

¿Pitan kanki? ¿Quién eres?

¿Imata ashillanki? ¿Qué busca usted?

¿Maypita kayanki? ¿De dónde son ustedes?

¿Imay yurirqayki? ¿Cuándo naciste?

¿Aykata munayan? ¿Cuánto quieres?

\subsection{Morfema interrogativo-disyuntivo}

En este caso, los dos elementos de la disyunción llevan el morfema - ku. La interrogación puede ser directa o indirecta.

¿Ishtiku, Kalluku rikechanan?

¿Esteban o Carlos debe despertarse? 
Maa, pikunash shiqiyanqa: kurukunakush, nuqakunakush.

A ver, quiénes van a ganar: los gusanos o nosotros.

El morfema -sh (después de vocal simple; -shi después de vocal larga y consonante) da el matiz semántico de que este discurso es dubitativo o no es tan propio del hablante que está repitiendo una expresión de otro u otros.

Kananqa, ¿puñkunkiku, watqankiku?

Ahora, ¿te vas a dormir o vas a vigilar?

Como se ve, se trata de oraciones interrogativas disyuntivas.

\subsection{Morfema hipocorístico}

El morfema, pospuesto al hipocorístico, sirve para expresar mayor trato afectivo.

Antuku (Antu-ku): Antonito

Mañuku (Mañu-ku): Manuelito

Shantaku (Shanta-ku): Santacrucito

Shatuku (Shatu-ku): Saturninito

Los hipocorísticos Antu, Mañu, Shanta, Shatu llevan el morfema -ku. Por la influencia del castellano también aparecen los femeninos Antuka, Mañuka, Shantaka, Shatuka (la apertura vocálica $-k u>-k_{0}$ trae su opuesto femenino terminado en $-a$ ), tal como hablan los bilingües.

Este morfema afectivo - ku también se usa para los nombres comunes. Añaku es el trato cariñoso para añas (zorrillo, mofeta). Muchos narradores del mito Achicay o Achcay o Achquee (Callejón de Huaylas) dicen añaku (zorrillito, mofetita), al referirse al animal que se compadeció y protegió a la desgraciada niña que, perseguida por la malvada Achicay, huía llevando en su bolsa los huesos de su hermanito devorado por la malvada vieja: Añaku, piñaskir, Achikaypa ñawinman ishpapuykun (El zorrillito, enojándose, orinó hacia los ojos de Achicay).

2. -ku, morfema verbal simple

2.1 Enfático. Puede significar alegría, lamento y énfasis sensorial o factual según el momento y la acción.

Qanyanpitana tamyakun.

Llueve ya desde ayer. 
Fuera de una enunciación afirmativa, hay más significados según las circunstancias: Es expresión de alegría si la lluvia es después de una sequía (la terrible usya que causa el caos en el campo); pero es un lamento si es durante los días de mucha lluvia. Los cambios de tono también sirven para diferenciar los mensajes.

Aswaykiqa ima alli mishkikun.

Tu chicha está muy deliciosa (Tu chicha está bien deliciosa).

Aquí se elogia la dulzura o la exquisitez de la bebida. La expresión «bien deliciosa» (alli mishkikun) es una forma de superlativo muy usada en Perú.

Hallqaqa alalaakunmi.

La jalca hace frío intenso.

Imanaachi, kananqa qilaakuumi.

No sé que me pasa, ocurre que hoy siento pereza.

Upa karchi, wañukuuman, ninki.

Seguramente por ser un tonto dices, ojalá me muera.

Como se ve en los ejemplos, las sensaciones pueden ser externas o internas.

2.2 Reflexivo. Hay verbos que aceptan la acción reflexiva.

Rirpuykichau rikaakurillaashaq.

Por favor, permítame verme en su espejo.

Lliqlipa rapranwan hampikullay.

Cúrese con la hoja de la hierbasanta.

Patsa waranqanyaq puñukushun.

Durmámonos hasta que amanezca.

El sustantivo patsa se refiere al mundo espacial y temporal.

2.3 Provisionativo. El sustantivo se verbaliza porque es el objeto de la búsqueda y del hallazgo.

Aku yantakuq. (yanta-ku-q).

Vayamos a buscar leña.

Kachikuqmi shamuu. (kachi-ku-q-mi)

Vengo a proveerme de sal.

Posiblemente, las siguientes expresiones han tenido el siguiente proceso de cambios: 
yantakuypaq $($ yanta $-k u-y-p a q)>$ yantakuq $($ sincopación de $-y-p a \ldots)$

leña -prov-inf -prop

kachikuypaq (kachi-ku-y-paq) > kachikuq (sincopación de -y-pa...)

sal -prov -inf - prop

prov: provisionativo; inf: infinitvo; prop: propósito

2.4 Ponerse alguna prenda de vestir. El nombre de la indumentaria para vestirse o usar se verbaliza con -ku, y significa el uso o la acción de ponerse.

Achay umata yanuptinmi tsukukuntsik. (tsuku-ku-ntsik.)

Nos ponemos el sombrero porque el sol sancocha nuestra cabeza.

tsuku: sombrero

$-k u$ : morf. verbalizador del nombre

-ntsik: morf. de primera persona plural que incluye al oyente

Unkukuyta munaptiipis, unkuu kapamaantsu. (unku-ku-y-ta)

Aunque quiera ponerme la túnica, qué mala suerte, no tengo mi túnica.

unku: túnica

$-k u$ : morf. verbalizador del nombre

$-y$ : morf. de infinitivo

-ta: morf. de objeto directo

Ullqum punchukun; warmim hakukun.

El varón usa el poncho; la mujer usa el manto. (El varón se emponcha; la mujer se enmanta)

punchu: poncho

-ku: morfema verbalizador del nombre

$-n$ : morfema de tercera persona

baku: manto

-ku: morf. verbalizador del nombre

$-n$ : morf. de tercera persona 
Los casos de 2.3, 2.4 son muestras y razones de por qué el bilingüe quechua-castellano verbaliza con mucha facilidad el nombre.

3. -ku, con morfema verbal compuesto

La distribución sintagmática del morfema - ku con otros morfemas verbales no es arbitraria. Esta combinación añade nuevos elementos al significado del tema verbal.

\subsection{Después de otro morfema verbal}

(1) - pa-ku: acción breve y no intencional. El morfema - ku está después del morfema temporal - $p a$ (indicador de que la acción es de duración breve). En esta estructura sintagmática el hablante expresa que la intención está ausente, o se supone ausente. Generalmente se refiere a hechos fortuitos.

Puyñta shumaq katay, ishkipakunkim. (ishki-pa-ku-nki-m)

Carga en tu espalda y con cuidado el cántaro, se te puede caer.

ishki: tema verbal de $i s h k i y=$ caer.

-pa: morf. de duración breve

$-k u$ : morf. de ausencia de intención

-nki: morf. de segunda persona verbal

$-m$ : morf. aseverativo en distribución postvocálica simple.

\section{Taqay warmish shullupakushqa (shullu-pa-ku-shqa)}

Dicen que aquella mujer ha abortado. (Se supone que este hecho es una desgracia).

shullu: tema verbal de shulluy $=$ parir

-pa: morf. de duración breve

-ku: morf. de ausencia de intención

-shqa: morf. de participio pasado, acción concluida

Wachapakuy (wacha-pa-ku-y)

Parir accidentalmente. Parir sin estar conviviendo.

wacha: tema verbal de wachay $=$ parir

-pa: morf. de duración breve

-ku: morf. de ausencia de intención

$-y$ : morf. de infinitivo 
El siguiente ejemplo es otro:

Allauchi, pachak watayuq karshi taqay aukisqa ishpapakun, ismapakun.

Pobrecito, dicen que aquel viejo se orina y se caga por tener cien años.

El ejemplo anterior, indudablemente, es un caso fortuito o desgracia.

La estructura de los verbos del ejemplo:

ishpa: tema verbal de $i$ shpay $=$ orinar

-pa: morf. de duración breve

$-k u$ : morf. de ausencia de intención

$-n$ : morf. de tercera persona verbal

isma: tema verbal de ismay = cagar, defecar

-pa: morf. de duración breve

$-k u$ : morf. de ausencia de intención

$-n$ : morf. de tercera persona verbal

En el caso de que la vocal final del morfema - pa llevara el cronema (fonema suprasegmental de duración) la nueva estructura es -paa-ku. Entonces, la acción es de más duración y con la participación de la voluntad. A continuación están los ejemplos con las vocales breves y largas, característica peculiar del quechua ancashino:

Waqtachau ishpapaakuy. (ishpa-pa-a-ku-y)

Orina afuera sin preocuparte de nada.

(Es la exhortación que se hace al menor que quiere escuchar o participar en la conversación de los mayores). Aquí se da el par mínimo: ishpapakuy (orinarse) / ishpapaakuy (orinar despreocupado sin importar el tiempo).

En los siguientes ejemplos se aprecian los significados diferentes por la ausencia o presencia del cronema.

Nuqaqa musyapakuqmi chaamui; manam musyapaakuqtsu.

Yo llego aquí para informarme; y no para criticar.

Otro par mínimo: musyapakuy (informarse) / muyapaakuy (criticar, hablar mal de otro).

(2) -ra-ku: énfasis en la acción de duración indefinida. Este sintagma compuesto está formado por -ra (morfema verbal de duración indefinida) y -ku (morfema enfático). El morfema temporal - ra, como en los ejemplos siguientes, puede llevar el cronema en la vocal a para enfatizar la acción duradera. 
Rikaraakuyllatsu; alli yachakuy. (rika-ra-a-ku-y-lla-tsu)

No te quedes solamente mirando; aprende bien.

Hitaraakuytsu; shaarir, imallatapis ruray. (bita-ra-a-ku-y-tsu)

No permanezcas echado; levantándote, haz siquiera algo.

(3) -ykacha-ku: énfasis en la acción repetida sin seriedad. Este sintagma está compuesto por $-y k a c h a$ (morfema verbal de acción repetitiva y sin ninguna seriedad) y $-k u$ (morfema enfático).

Takita, wiyaskir patsa, sikita milluykachakunki; tushu pacha kanki. (millu-ykacha-ku-nki)

Apenas oyendo la música contorneas tu cadera; eres un vicioso del baile.

millu: tema verbal de milluy $=$ contornear

-ykacha: morf. de acción repetida sin seriedad

-ku: morf. enfático

$-n k i$ : morf. de segunda persona verbal

Qipillaata yataykachakuytsu. (yata-ykacha-ku-y-tsu)

Por favor, no manosee mi bulto.

yata: tema verbal de yatay $=$ manosear, palpar, tocar

-ykacha: morf. de acción repetida sin seriedad

-ku: morf. enfático

$-y$ : morf. de imperativo en segunda persona

-tsu: morfema negativo

En el castellano el morfema -ear del infinitivo sirve, generalmente, para expresar la acción repetitiva. «Este sufijo es uno de los más activos en la derivación verbal en todas las variedades del español, particularmente en las americanas». «Expresan el ejercicio reiterado de una actividad» (NGLE, 2010, 8.3.1; 8.3.1f).

(4) -taq-ku: sorpresa negativa. Esta estructura del morfema aspectual -taq (ocurre que, sorpresa) con -ku (enfático) expresa la idea negativa. Y puede estar después del nombre, pronombre, adjetivo, adverbio y verbo.

Después del nombre

Qapaqtaqku kanki. (qapaq-taq-ku)

Date cuenta, no eres dios.

Después del pronombre 
Nuqataqku. (nuqa-taq-ku)

Ocurre que yo no soy.

Después del adjetivo

Allitaqku. (alli-taq-ku)

Ocurre que no es bueno.

Después del adverbio

Qanyantaqku rikarqaq, qanyaatinmi. (qanyan-taq-ku)

Me consta que no fue ayer cuando te vi, fue anteayer.

Después del verbo

Wamraykiqa rikchantaqku. (rikecha-n-taq-ku)

Ocurre que tu criatura no se despierta.

rikecha: tema verbal de rikechay $=$ despertar

$-n$ : morf. de tercera persona verbal

-taq: morf. de sorpresa

-ku: morf. Enfático

En este caso los morfemas -taq-ku van pospuestos al verbo ya conjugado por eso no se puede decir que se trata, propiamente, de morfemas verbales.

(5) -tsi-ku: acción en beneficio o perjuicio del sujeto. Este sintagma está compuesto por el morfema causativo -tsi y el enfático - ku; ambos expresan que la acción es en beneficio o daño del sujeto.

Taqay aukisqa, mana qillqata riqirmi, qillqatsikullan. (qillqa-tsi-ku-lla-n)

Aquel anciano pide que le hagan el favor de escribir porque no conoce el alfabeto.

qillqa: tema verbal de qillqay $=$ escribir

-tsi: morf. verbal causativo

-ku: morf. enfático

-lla: morf. de cortesía

$-n$ : morf. de tercera persona verbal

Qillayniki kanqanyaq alli hampitsikuy. (hampi-tsi-ku-y)

Hazte curar bien mientras tengas dinero.

hampi: tema verbal de hampiy $=$ curar, remediar 
-tsi: morf. verbal causativo

$-k u$ : morf. enfático

$-y$ : morf. de imperativo en segunda persona

(6) -tsa-ku: transformación enfática. Estructura formada por el morfema transformativo -tsa y el morfema enfático - ku ; ambos expresan la transformación con énfasis. Si la vocal a (margen final del morfema transformativo) es de duración larga expresa mayor énfasis.

Yaqashqa awatam allitsakuu. (alli-tsa-ku-u)

Arreglo con dedicación el tejido dañado.

Pukru rimaq pampata pampatsaakushun. (pampa-tsa-a-ku-shun)

Aplanemos con esmero la plaza hundida.

\subsection{Antes de otro morfema verbal}

En esta estructura hay dos casos:

(1) -ku-ri: Combinación de énfasis e incoacción. El morfema -ku precede al morfema incoativo $-r i$. El tema verbal se enriquece con los semas enfático e incoativo.

Chukru tantallapis mikukurishun. (miku-ku-ri-shun)

Comamos con gusto aunque sea el pan duro.

miku: tema verbal de mikuy $=$ comer

-ku: morf. enfático

-ri: morf. incoativo

-shun: morf. de imperativo o exhortativo de primera persona del plural

Pichi uraykaamun; maa, riqikurillay. (riqi-ku-ri-lla-y)

Alguien está bajando hacia aquí; a ver, reconózcalo, por favor.

riqi: tema verbal de riqiy $=$ reconocer

-ku: morf. enfático

-ri: morf. incoativo

-lla: morf. de cortesía

$-y$ : morf. de imperativo de segunda persona 
(2) -ku-yka: Acción con énfasis y en proceso de realización. El morfema enfático -ku precede al morfema progresivo - yka. El resultado es enfático y progresivo.

Qishyakuykaptii chaaramunki. (qishya-ku-yka-pti-i)

Llegas cuando estoy sufriendo por la enfermedad.

qishya: tema verbal de qishyay = enfermar, estar enfermo

-ku: morf. enfático

-yka: morf. verbal progresivo

-pti: morf. subordinador temporal

La vocal larga (ii) del margen final de la sílaba - pti es la marca verbal de la primera persona del singular.

Huchushqa rarqatam pirqakuykaa. (pirqa-ku-yka-a)

Estoy levantado el muro de la acequia derrumbada.

\section{CONCLUSIONES}

El variado uso del morfema - ku enriquece el discurso; pero, al mismo tiempo, crea dificultades a los que aprenden el quechua como segunda lengua.

Los quechuahablantes para expresar los hipocorísticos, aunque los nombres sean ajenos a su lengua, recurren a la palatalización: Shanti (Santiago), Ishti (Esteban), Kallu (Carlos), Mañu (Manuel, Manuela).

La frecuente y fácil verbalización de los nombres es una característica del quechua. Y los bilingües quechua-castellano siguen con esta tendencia.

\section{REFERENCIAS BIBLIOGRÁFICAS}

Carranza, Francisco. 2002 La cantidad vocálica en el quechua de Áncash. Lima: Homenaje a Luis Jaime Cisneros, Tomo I, Pontificia Universidad Católica del Perú.

Carranza, Francisco. 2003. Diccionario del quechua ancashino - castellano. Frankfurt, Vervuert.

Julca, Félix. 2009. Quechua ancashino: una mirada actual. Lima: Fondo Editorial del Pedagógico San Marcos.

Ortiz, Rescaniere. 1992. El quechua y el aymara. Madrid: Colecciones MAPFRE.

Quilis, Antonio. 1981. Fonética acústica de la lengua española. Madrid: Gredos.

Quilis, Antonio y Fernández, Joseph. 1975. Curso de fonética y fonología españolas. Madrid: Consejo Superior de Investigaciones Científicas. 
| Francisco Carranza

Real Academia Española. 1973. Esbozo de una nueva gramática de la lengua española. Madrid: Espasa Calpe. .

Real Academia Española. 2005. Diccionario panbispánico de dudas. Colombia: Santillán.

Real Academia Española. 2010. Nueva gramática de la lengua española, (NGLE). Colombia: Planeta.

Fecha de recepción: 23 de febrero 2016

Fecha de aceptación: 26 de mayo 2016

\section{Correspondencia:}

Francisco Carranza Romero

carranzako@gmail.com 